\title{
New NCCN Guidelines: Recognition and Management of Immunotherapy-Related Toxicity
}

\author{
Presented by John A. Thompson, MD
}

\section{Abstract}

Immune checkpoint inhibitors (ICls) are now FDA-approved for the treatment of 8 different cancers, and more approvals are likely, including use of these drugs in combinations. Although ICls represent a true advance in cancer care, they can cause a range of immune-related adverse events. As more experience with $\mathrm{ICls}$ is gained, more information is becoming available on immunotoxicity and optimal management. Physicians and patients need to be educated about potential adverse events and management of ICl-associated toxicity. In recognition of the need for better information, NCCN in collaboration with ASCO has developed the first set of NCCN Guidelines for Management of Immunotherapy-Related Toxicities.

J Natl Compr Canc Netw 2018;16(5.5):594-596 doi: 10.6004/jncen.2018.0047

Immune checkpoint inhibitors (ICIs) have revolutionized cancer treatment for the $25 \%$ of patients who experience response. ICIs are now FDA-approved for the treatment of 8 different cancers.

"Along with the good news, we have seen an emergence of a spectrum of immune-related adverse events [AEs]. The common mechanism is that when these drugs 'release the brakes' on the immune system, then the immune system can also attack normal tissue," said John A. Thompson, MD, Professor of Medicine, University of Washington; Co-Director, Melanoma Clinic, Seattle Cancer Care Alliance; and Chair of the NCCN Guidelines Panel for Management of ImmunotherapyRelated Toxicities.

NCCN in collaboration with ASCO developed a standardized approach for the recognition, management, and education of ICI-related toxicity. ASCO published its Practice Guideline in the Journal of Clinical Oncology on February 14, 2018, ${ }^{1}$ with NCCN releasing theirs the same day (available at NCCN.org). At the 23rd NCCN Annual Conference, Dr. Thompson presented key points from the new NCCN Guidelines.

Presented by John A. Thompson, MD, Seattle Cancer Care Alliance, Seattle, Washington.

Dr. Thompson has disclosed that he has served as a scientific advisor for Calithera and Celldex.

Correspondence: John A. Thompson, MD, Seattle Cancer Care Alliance, 825 Eastlake Avenue East, Mailstop CE2-110, Seattle, WA 98109.

Email: jat@uw.edu
Dr. Thompson emphasized that the NCCN Guidelines are based on expert opinion and there are no prospective trials on treatment of immunotherapy-related AEs. He pointed out that grades 3-5 immunotherapyrelated AEs occur with greater frequency with CTLA-4 inhibitors than with PD-1 or PD-L1 inhibitors. Toxicity is also more severe with combinations of CTLA-4 with PD-1 or PD-L1 inhibitors than with either class of agent alone. "Physicians have to be alert for these effects, and we have to educate our patients to be prepared," he stated.

The pattern of onset of immunotherapy-related toxicity varies. Some toxicities may occur early, some later, and some even after completion of treatment, he noted. "In general, when a patient encounters a serious toxicity, the checkpoint inhibitor should be stopped until the toxicity resolves. Patients may fear that they will lose the antitumor effect, but studies of outcomes in patients who discontinue therapy due to AEs show no statistically significant difference in progression-free and overall survival. This provides some reassurance that stopping therapy due to AEs does not jeopardize the anticancer effect of immunotherapy," Dr. Thompson elaborated.

ICI therapy is effective in approximately $25 \%$ of patients, and the drugs are expensive, so research is being conducted to find a biomarker for response. Thus far, results have been limited. Recent studies suggest that tumor mutational burden plus PD-L1 expression may select patients for therapy with an anti-PD-1 or anti-PD-L1 drug. The presence of high tumor mutation burden and high PD-L1 expression suggest that durable clinical benefit is more likely (Figure 1$){ }^{2}$ 


\section{Dermatologic Toxicity}

Maculopapular rash, which can be severe, is one of the more common toxicities seen with ICI therapy. Other dermatologic toxicities can include pruritus, bullous dermatoses, vasculitis, and vitiligo. Management of dermatologic and other AEs is according to grade of severity, Dr. Thompson said. For grade 3-4 maculopapular rash or pruritus, immunotherapy should be withheld and high-dose steroids should be given.

Bullous dermatoses and Stevens-Johnson syndrome or toxic epidermal necrolysis may be severe and life-threatening, and require urgent dermatologic consultation for skin biopsy and inpatient treatment with steroids. The ICI should be discontinued permanently.

\section{Gastrointestinal Toxicity}

Gastrointestinal toxicity encountered with ICI therapy can include diarrhea and colitis. Patients who experience either of these AEs may still achieve a durable complete response, Dr. Thompson noted.

For moderate to severe toxicity, a stool sample should be obtained to rule out infectious etiology,

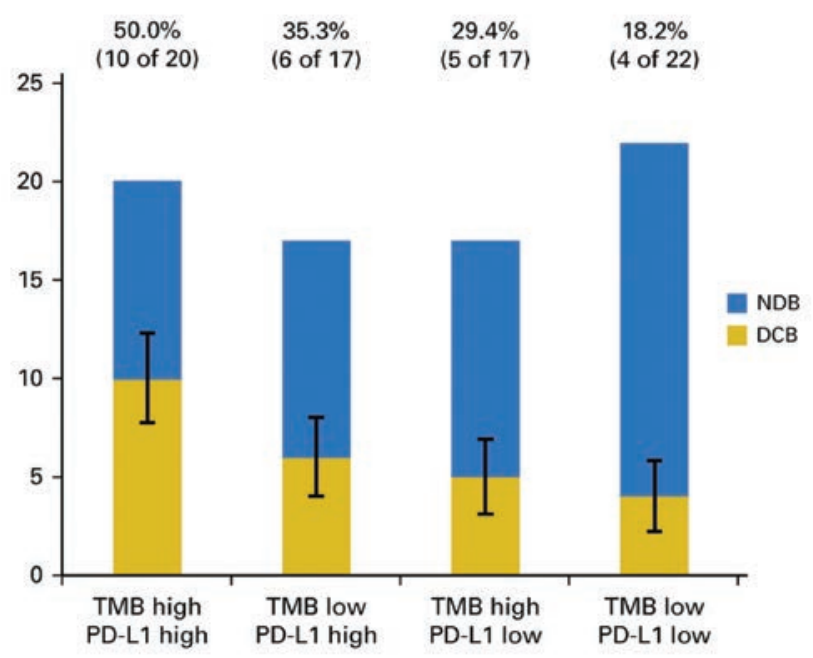

Figure 1. Molecular determinants of response to PD-1 and PD-L1 blockade in patients with non-small cell lung cancer. A total of 240 patients were treated with anti-PD-1 or PD-L1, and had TMB profiled by targeted next-generation sequencing, of which 84 had PD-L1 immunohistochemistry performed.

Abbreviations: DCB, durable clinical benefit; NDB, no durable benefit; TMB, tumor mutational burden.

From Rizvi H, Sanchez-Vega F, La K, et al. Molecular determinants of response to anti-programmed cell death (PD)-1 and anti-programmed cell death-ligand 1 (PD-L1) blockade in patients with non-small-cell lung cancer profiled with targeted next-generation sequencing. Reprinted with permission. (c) 2018 All rights reserved. Rizvi H, et al: J Clin Oncol 2018;36:633-641. and a gastrointestinal consultation is recommended. ICIs should be withheld for moderate gastrointestinal toxicity and discontinued for severe cases. Intravenous steroids are recommended. "Occasionally steroids don't control gastrointestinal AEs. The NCCN Guidelines recommend infliximab, which may successfully treat the colitis," he explained.

\section{Liver Toxicity}

For mild liver toxicity, immunotherapy can be continued and the patient should be monitored with increased frequency. For moderate toxicity, immunotherapy should be withheld, the patient should be monitored, and steroids should be considered if liver function test results worsen.

For severe and life-threatening liver toxicity, ICI therapy should be permanently discontinued, inpatient care should be considered, and infliximab should not be used. If steroids are not effective, mycophenolate mofetil can be used in the second line to control hepatitis.

"Patients are concerned about whether they can get re-treatment," Dr. Thompson noted. A retrospective study of 80 patients who discontinued combination therapy with a CTLA-4 inhibitor and a checkpoint inhibitor due to AEs examined the safety and risks associated with reintroduction of an anti-PD-1 agent. $^{3}$

\section{Pancreas Toxicity}

Pancreatic AEs may manifest as asymptomatic elevations in amylase and/or lipase levels. "For the most part, asymptomatic elevations do not require withholding ICIs. For persistent elevations, be sure to rule out subclinical pancreatitis," Dr. Thompson said.

For acute pancreatitis that is moderate, immunotherapy should be held and high-dose steroids initiated with a planned 6-week taper. If severe, immunotherapy should be discontinued and the patient treated with steroids.

\section{Endocrine Toxicity}

New-onset hyperglycemia is a rare but concerning AE in patients on ICI therapy. For new-onset type I diabetes, patients should be evaluated for diabetic ketoacidosis (DKA). If present, immunotherapy 
should be held and inpatient care with an endocrine consultation should be initiated. If DKA is not present, immunotherapy may be continued and blood glucose levels should be monitored with each dose. Patients should also be advised to modify their diet and lifestyle accordingly. Endocrine consultation should be considered if symptoms persist and if glucose levels remain uncontrolled.

Thyrotoxicosis may also occur with immunotherapy. Beta-blocker therapy may be given until symptoms resolve.

Adrenal insufficiency has also been reported in patients on ICI therapy. "This can come on insidiously and be accompanied by thyroid or other endocrine toxicities," Dr. Thompson explained. "It is important to make the diagnosis and start the patient on adrenal hormone replacement before other hormonal therapy to avoid adrenal crisis."

Hypophysitis is relatively common and sometimes presents without headaches. If the patient is symptomatic, prednisone should be considered. Immunotherapy should be withheld until symptoms resolve, and hormone replacement should be given if indicated.

"Pituitary dysfunction is usually not reversible," he noted. "Prolonged physiological replacement therapy may be required."

\section{Lung Toxicity}

Pneumonitis can occur and can be mild, moderate, or severe. Immunotherapy should be held for mild and moderate hypophysitis, and patients should be reassessed every 1 to 2 weeks.

For severe pneumonitis, discontinuation of immunotherapy is required and inpatient care is advised. Patients should also undergo a workup for infections. High-dose steroids are advised with a taper over 6 weeks or longer. If there is no improvement after 48 hours, infliximab, mycophenolate mofetil, or intravenous immunoglobulin should be considered.

\section{Other Toxicities}

The toxicities described are the tip of the iceberg. Ocular toxicity, a host of neurologic toxicities (eg, myositis, peripheral neuropathy, encephalitis, meningitis, transverse myelitis), and cardiac toxicities can all occur. Clinicians should be alert for these effects and consult the NCCN Guidelines when needed.

"For patients with a relatively good prognosis related to their cancer, an assessment of risks/benefits of ICI therapy is especially important. For those patients on immunotherapy, physicians should have a high level of suspicion and tell their patients and caregivers what symptoms to look for. Immunotherapy should be held for most grade 2 AEs. Stop immunotherapy for $\geq$ grade 3 AEs and high-dose steroids should be initiated. When toxicity reverts to $\leq$ grade 1 , resumption may be offered but caution is advised, especially in patients with early-onset AEs. Caution should be exercised when considering immunotherapy in a patient with current or previous autoimmune disease. Take a thorough family history and check for autoimmune disease," Dr. Thompson stated.

\section{References}

1. Brahmer JR, Lacchetti C, Schneider BJ, et al. Management of immunerelated adverse events in patients treated with immune checkpoint inhibitor therapy: American Society of Clinical Oncology Practice Guideline [published online February 14, 2018]. J Clin Oncol. doi: 10.1200/JCO.2017.77.6385

2. Rizvi H, Sanchez-Vega F, La K, et al. Molecular determinants of response to anti-programmed cell death (PD)-1 and anti-programmed cell death-ligand

1 (PD-L1) blockade in patients with non-small-cell lung cancer profiled with targeted next-generation sequencing. J Clin Oncol 2018;36:633-641.

3. Pollack $\mathrm{MH}$, Betof $\mathrm{A}$, Dearden $\mathrm{H}$, et al. Safety of resuming anti-PD-1 in patients with immune-related adverse events (irAEs) during combined anti-CTLA-4 and anti-PD1 in metastatic melanoma. Ann Oncol 2018;29:250-255. 